\title{
Polyphase Analysis of Subband Adaptive Filters
}

\author{
Stephan Weiss ${ }^{1}$. Robert W. Stewart ${ }^{2}$, Moritz Harteneck ${ }^{3}$, and Alexander Stenger \\ ${ }^{1}$ Dept. Electronics \& Computer Science, University of Southampton, UK \\ ${ }^{2}$ Dept. Electronic \& Electrical Eng., University of Strathclyde, Glasgow, UK \\ ${ }^{3}$ Infineon Technologies AG, Munich, Germany \\ Telecommunications Institute I, University of Erlangen, Germany \\ s.weissQecs.soton.ac.uk, r.stewartQeee.strath.ac.uk
}

\begin{abstract}
Based on a polyphase analysis of a subband adaptive filter (SAF) system, it is possible to calculate the optimum subband impulse responses to which the SAF system will converge. In this paper, we give some insight into how these optimum impulse responses are calculated, and discuss two applications of our technique. Firstly, the performance limitations of an SAF system can be explored with respect to the minimum mean square error performance. Secondly, fullband impulse responses can be correctly projected into the subband domain, which is required for example for translating constraints for subband adaptive beamforming. Examples for both applications are presented.
\end{abstract}

\section{Introduction}

Adaptive filtering in subbands is a popular approach to a number of problems, where high computational cost and slow convergence due to long filters permits the direct implementation of a fullband algorithm. These problems include acoustic echo cancellation $[5, \overline{3}]$. identification of room acoustics [8], equalization of acoustics [10], or beamforming [6, 11]. In Fig. 1, a subband adaptive filter (SAF) is shown in a system identification setup of an unknown system $s[n]$, whereby the input $x[n]$ and the desired signal $d[n]$ are split into $K$ frequency bands by analysis filter banks built of bandpass filters $h_{k}[\Omega]$. Assuming a cross-band free SAF design [3], an adaptive filter $w_{k}[n]$ is applied to each subband decimated by $N \leq K$. Finally, the fullband error signal $e[n]$ can be reconstructed via a synthesis bank.

However, subband adaptive filters (SAF) are sub- ject to a number of limitations, which have been investigated, for example, with respect to the required filter length [3.14] or to lower bounds for the MMSE and the modelling accuracy [12]. These analyses have been performed using modulation description $[3,7]$, time domain [14], or frequency domain approaches [5, 12].

Here, we discuss the SAF in Fig. 1 using a polyphase description of the signals and filters therein [2]. This will provide some new and alternative insight into the optimality of SAFs. Sec. 2 analyses the subband errors, which leads to the derivation and discussion of an optimal subband filter structure in Sec. 3. Application examples for the proposed techniques are underlined by simulations in Sec. 4 .

\section{Polyphase Analysis of Subband Errors}

The aim of this section is to express the subband error signals, $E_{k}^{d}(z) \bullet-\circ e_{k}(z)$, in terms of the polyphase components of all involved signals and systems. Implicitly, this means that we are trying to find a linear, time-invariant (LTI) description of the error signal. To achieve this task, we first require suitable representations for the decimated desired signal in the $k$ th subband, $D_{k}^{d}(z) \bullet \cdots d_{k}[n]$, and for the decimated input signal in the $k$ th subband, $X_{k}^{d}(z) \bullet \cdots x_{k}[n]$, as labelled in Fig. 1. In our notation, superscript $\{\cdot\}^{d}$ for z-transforms of signals refers to decimated quantities, while normal variables such as $X_{k}(z)$ indicate undecimated signals, i.e. in this case the input signal in the $k$ th subband before going into the decimator as shown in Fig. 1.

The formulation of the $k$ th decimated desired signal $D_{k}^{d}(z) \bullet-\circ d_{k}[n]$ will be the first aim. We define the expansion of the desired signal $D(z) \bullet-\circ d[n]$ into 


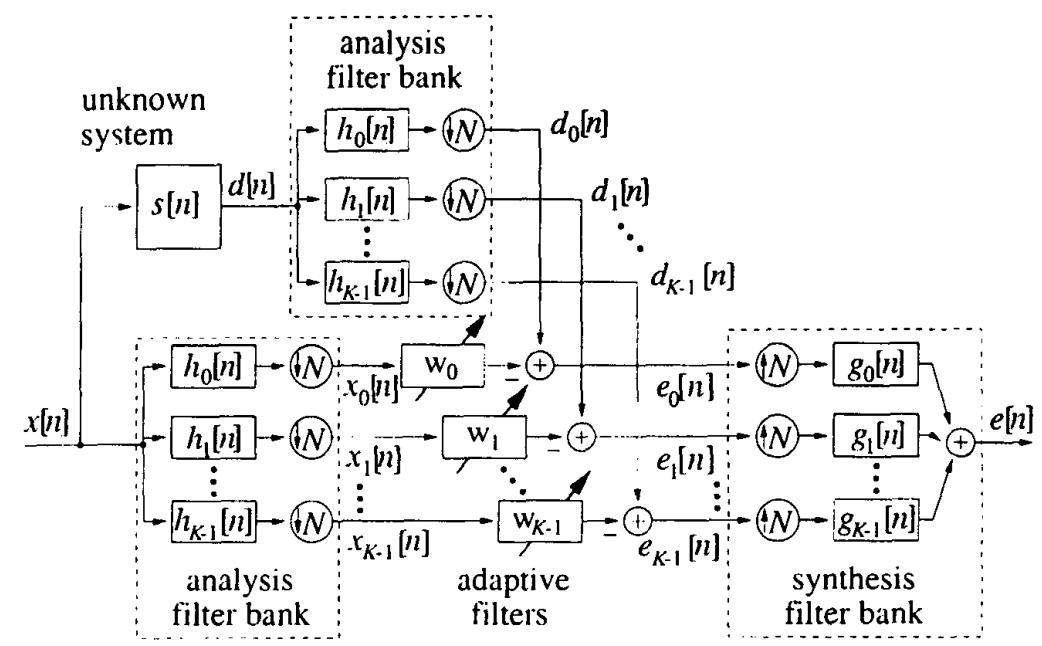

Fig. 1. Subband adaptive filter (SAF) in a system identification setup.

type-2-polyphase componerts [9] $D_{n}(z)$,

$$
D(z)=\sum_{n=0}^{N-1} z^{-N+n+1} \cdot D_{n}\left(z^{N}\right),
$$

and a type-1-polyphase expansion [9] of the analysis filters $\ddot{H}_{k}(\dot{z})$,

$$
H_{k}(z)=\sum_{n=0}^{N-1} z^{-n} \cdot H_{k, n}\left(z^{N}\right) .
$$

Similarly, for all following polyphase expansions, it is assumed for compatibility that systems are represented by a type-1-polyphase expansion, and signals by type-2-polyphase expansions. Bringing these polyphase components of (1) and (2) into vector form,

$$
\begin{aligned}
\underline{D}(z) & =\left[\begin{array}{llll}
D_{0}(z) & D_{1}(z) & \cdots & D_{N-1}(z)
\end{array}\right]^{T} \\
\underline{H}_{k}(z) & =\left[\begin{array}{lllll}
H_{k \mid 0}(z) & H_{k \mid 1} & \cdots & H_{k \mid N-1}(z)
\end{array}\right]^{T}
\end{aligned}
$$

$D_{k}^{d}(z)$ can now be expressed as

$$
D_{k}^{d}(z)=\underline{H}_{k}^{T}(z) \cdot \underline{D}(z) .
$$

To trace the desired signal back to the input signal $X(z) \cdot-\circ x[n]$, the expression $D(z)=S(z) \cdot X(z)$ can be appropriately expanded such that the $n$th polyphase component in (3) may be written as

$$
D_{n}(z)=\underline{S}^{T}(z) \cdot \Lambda_{n}(z) \cdot \underline{X}(z)
$$

The vector $\underline{S}(z)$ contains the type-1-polyphase components of the unknown system $S(z) \bullet-\circ s[n]$; while
$X(z)$ is defined similarly to (3) based on the type-2-polyphase components of the input signal $X(z) \bullet-\circ x[n]$. The matrix $\boldsymbol{\Lambda}_{n}(z)$ in (6) is a delay matrix defined as

$$
\boldsymbol{\Lambda}_{n}(z)=\left[\begin{array}{cc}
\mathbf{0} & \mathbf{I}_{N-n} \\
z^{-1} \mathbf{I}_{n} & \mathbf{0}
\end{array}\right]
$$

With (5) and (6), the decimated $k$ th desired subband signal $D_{k}^{d}(z)$

$$
D_{k}^{d}(z)=\underline{H}_{k}^{T}(z)\left[\begin{array}{c}
\underline{S}^{T}(z) \Lambda_{0}(z) \\
\underline{S}^{T}(z) \Lambda_{1}(z) \\
\vdots \\
\underline{S}^{T}(z) \Lambda_{N-1}(z)
\end{array}\right] \underline{X}(z)=\underline{H}_{k}^{T}(z) \mathbf{S}(z) \underline{X}(z)
$$

can be assembled. For brevity, the substituted matrix $\mathbf{S}(z)$ holds differently delayed polyphase components of the unknown system.

With the type-2-polyphase components of $X(z)$ and the polyphase representation of the analysis filter bank in (2) it is comparably simple to derive the $k$ th decimated input signal $X_{k}^{d}(z)$ as

$$
X_{k}^{d}(z)=\underline{H}_{k}^{T}(z) \cdot \underline{X}(z) .
$$

Finally, with (8), (9), and the transfer function of the $k$ th adaptive filter $W_{k}^{*}(z) \bullet \circ w_{k}[n]$ it is possible to formulate the $k$ th subband error signal, $E_{k}^{d}(z) \bullet-\circ e_{k}[n]$ :

$$
\begin{aligned}
E_{k}^{d}(z) & =D_{k}^{d}(z)-W_{k}(z) \cdot X_{k}^{d}(z) \\
& =\left\{\underline{H}_{k}^{T}(z) \cdot \mathbf{S}(z)-\underline{H}_{k}^{T}(z) \cdot W_{k}(z)\right\} \underline{X}(z)(11)
\end{aligned}
$$




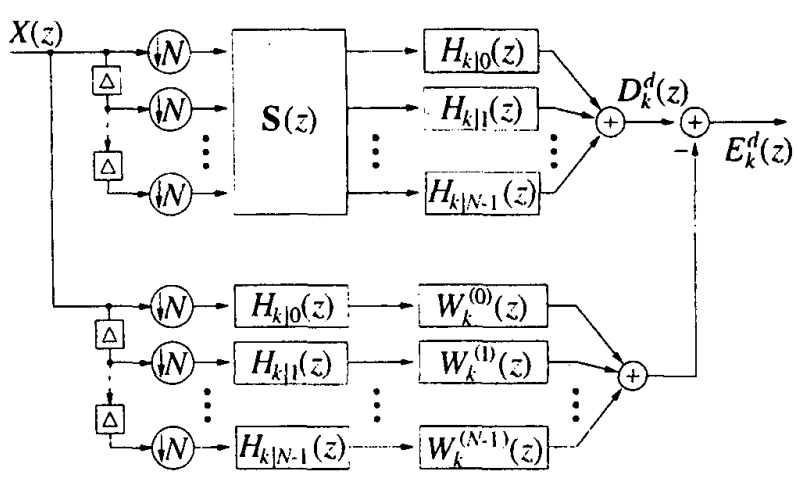

Fig. 2. SAF optimal polyphase solutions in the $k$ th subband.

Note, that for the description of $E_{k}^{d}(z)$, the timerarying decimators have been swapped with all system elements in the SAF structure of Fig. 1, and (11) only contains LTI terms.

\section{Subband Error Minimization}

This section discusses the optimum subband filters to solve the identification problem outlined in Sec. 1, based on the polyphase analysis of the subband errors in the previous Sec. 2.

\subsection{Optimum Subband Filters}

As no external disturbance of the SAF system in Fig. 1 by observation noise is present, ideally the attainable minimum mean square error (MMSE) should be zero. This is identical to setting $E_{k}^{d}(z)$ in (11) equal to zero. As independence of the optimum solution from the input signal's polyphase components in $\underline{X}(z)$ is desirable, the requirement for optimality (in every sense) is given by

$$
\cdot \underline{H}_{k}^{T}(z) \cdot \mathbf{S}(z) \stackrel{!}{=} \underline{H}_{k}^{T} \cdot W_{k, o p t}(z) \text {. }
$$

Hence, we obtain $N$ cancellation conditions indicated by superscripts $\{\cdot\}^{(n)}$, which have to be fulfilled:

$W_{k, \text { opt }}^{(n)}(z)=\frac{\underline{H}_{k}^{T}(z) \cdot \Lambda_{n}^{T}(z) \cdot \underline{S}(z)}{H_{k \mid n}(z)} \quad \forall n \in\{0 ; N-1\}$.

Therefore, ideally $W_{k}(z)$ in (11) and (12) should be replaced by an $N \times N$ diagonal matrix with entries $W_{k}^{(n)}(z), n=0(1) N-1$. For the $k$ th subband, this solution with $N$ polyphase filters is depicted in Fig. 2.

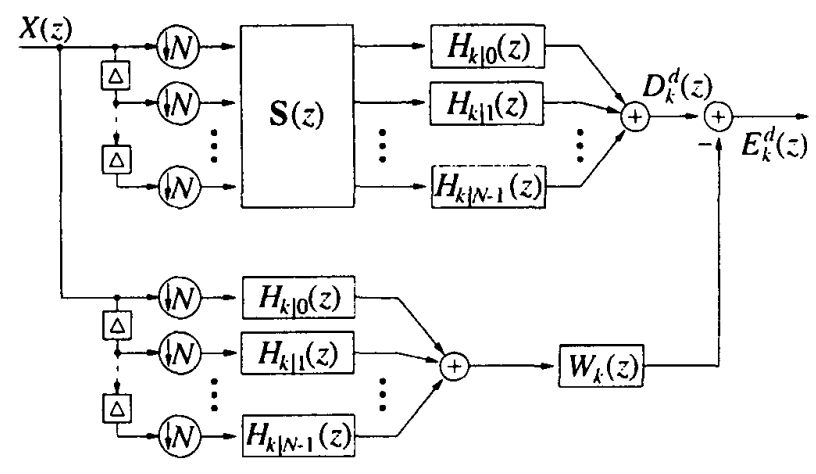

Fig. 3. SAF standard solution in the $k$ th subband.

\subsection{Interpretation}

Alternatively, the $n$th optimum solution can be written as

$$
W_{k, \mathrm{opt}}^{(n)}(z)=\sum_{\nu=0}^{N-1} A_{k \mid n}^{(\nu)}(z) \cdot S_{\nu}(z)
$$

and interpreted as a superposition of polyphase components $S_{\nu}(z)$ of the unknown system $S(z)$, "weighted" by transfer functions

$$
A_{k \mid n}^{(\nu)}(z)=z^{-\lfloor(n+\nu) / N\rfloor} \cdot \frac{H_{k \mid(n+\nu) \bmod N}(z)}{H_{k \mid n}(z)} .
$$

From this, we can observe, that the length of the optimum subband responses is obviously given by $1 / N$ of the order of $S(z)$, but extended by the transfer functions (15). These extending transients are causal for poles of $A_{k \mid n}^{(\nu)}(z)$ within the unit circle, and acausal for stabilized poles outside the unit-circle [13], motivating a non-causal optimum response.

Further, for an ideal, alias-free filter bank, the polyphase components $H_{k \mid n}(z)$ in (15) should not differ in magnitude but only in phase, which is compensated for by the delay element in (15). Hence all $N$ solutions become identical, an the $N$ optimum polyphase filters can be replaced by a single filter $W_{k \text {.opt }}(z)$ as shown in Fig. 3, which is equivalent to the original standard setup in Fig. 1. In general, and particularly if aliasing is present, the optimum polyphase solutions $W_{k, \mathrm{opt}}^{(n)}(z)$ will differ. In this case the optimum standard SAF solution according to Fig. 3 gives the closest $l_{2}$ match to all $N$ polyphase solutions:

$$
W_{k, \text { opt }}(z)=\frac{1}{N} \sum_{n=0}^{N-1} W_{k, \mathrm{opt}}^{(n)}(z)
$$




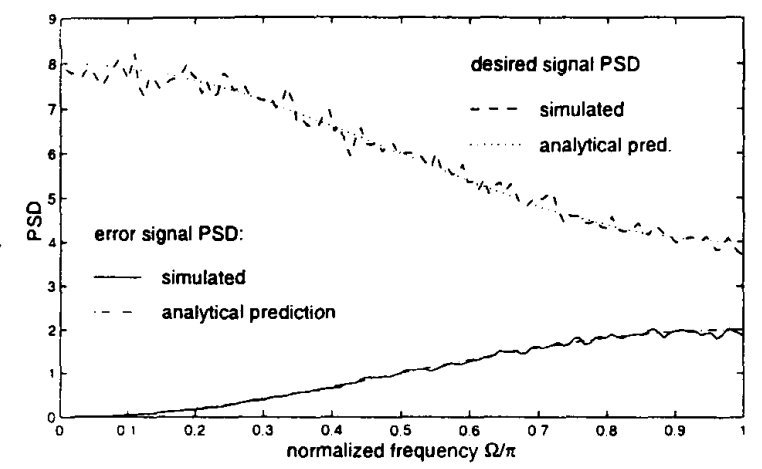

Fig. 4. Comparison between simulated and analytically predicted PSDs in the Oth subband.

The error made in this approximation explains error and modelling limitations of the SAF approach and represents an alternative coefficient / time-domain description as opposed to spectrally motivated SAF error explanations in the literature [3. 12]. Interestingly; in [7] the same polyphase structure as in Fig. 2 is obtained using modulation description [2] 5, although only for the critically sampled case.

\section{Applications and Simulations}

We now want to explore two applications for the polyphase analysis presented in Secs. 2 and 3.

\subsection{Error Limits}

A very basic example given in the following will demonstrate the ability of the proposed analysis to predict optimal subband responses and error terms in the context of SAF systems. For this example, a 2-channel critically decimated standard SAF system as in Fig. 1 based on a Haar filter bank [2] should adaptive identify an unknown system $S(z)=1+z^{1}$, using unit variance Gaussian white noise excitation. Looking at the channel $\bar{k}=0$ produced by the Haar lowpass filter $H_{0}(z)=1+z^{-1}$, an RLS adaptive algorithm [4] converges to the solution

$$
W_{0, \text { adapt }}(z)=1.4873+0.5067 z^{-1} \text {. }
$$

Analytical evaluation via (14) and (15) yields the $N=2$ optimum polyphase solutions for the band $k=0$

$$
W_{0, \mathrm{opt}}^{-(0)}(z)=2, \quad W_{0 . \mathrm{pt}}^{-(1)}(z)=1+z^{-1},
$$

which refers to the optimal subband adaptive filter structure shown in Fig. 2. If this setup is simplified to the structure of the standard SAF system in Fig. 3, the analytical solution (16) calculated from (18) is given by the mean of the two optimum polyphase solutions,

$$
W_{0 . \mathrm{opt}}(z)=1.5+0.5 z^{-1} \text {. }
$$

This result obviously very closely agrees with the simulation result in (17).

Based on the above analytical solutions, it is now possible to predict the subband error signal as due to the mismatch of (18) and (4.1). The PSD of the 0th adapted subband error signal, $S_{e_{0}}\left(e^{j \Omega}\right)$, can be analytically predicted by inserting the optimum standard solution (16) into (11).

$$
S_{e_{0}}\left(e^{j \Omega}\right)=\left|E_{k}^{d}\left(e^{j \Omega}\right)\right|^{2}=1-\cos \Omega
$$

which can be used to determine the minimum mean squared error of the SAF system alternative to spectral methods [12]. Fig. 4 demonstrates the excellent fit between the analytically calculated PSD in (19), and the measured results from the RLS simulation. Also shown is the analytically predicted and measured PSD of the 0 th desired subband signal $S_{d_{0}}\left(e^{j \Omega}\right)=6+2 \cos \Omega$ (hence the uncancelled error signal) calculated via (5).

\subsection{Subband Projection}

A second application example is concerned with substituting subband adaptive system identification with the proposed analysis. If a digital impulse response is given in the fullband, but should be projected into the subband domain, an SAF identification is mostly required. This could be to produce computationally efficient sound processing from a given (fullband) room transfer function [8], or the projection of constraints into the subband domain when performing subband adaptive beamforming [11].

We assume an SAF system with $K=8$ channels decimated by $N=6$, and wide analysis filters to improve spectral whitening in the subbands [1]. Analysis and synthesis banks are derived from the two different prototype filters shown in Fig. 5. With a lowpass fullband response $s[n]$ given, an RLS adaptive identification yields in the subband $k=0$ the coefficients shown in Fig. 6, along with the analytic solution according to (14) and (16). For the analytic solution, the roots of the denominator polynomial in (15) have been substituted by appropriate causal and a-causal FIR filters. Obviously, the match between adaptive and analytical solution is very close, and themore direct analytical approach can replace an adaptive projection. 

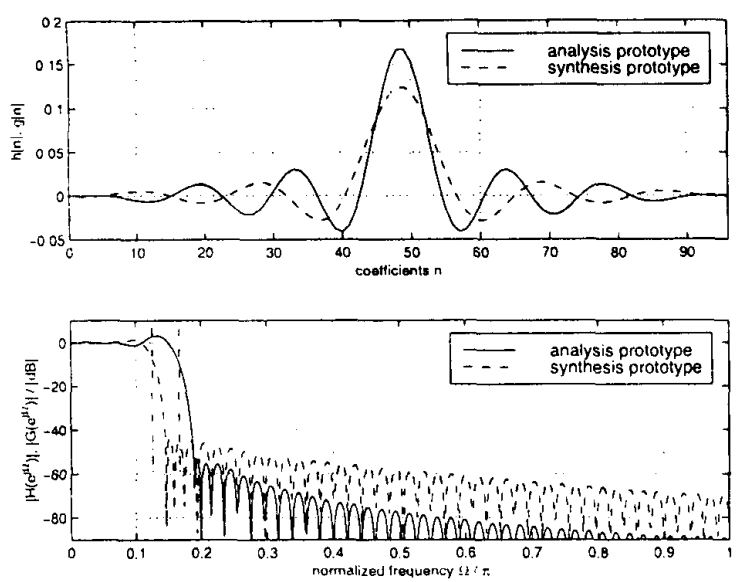

Fig. 5. Prototype filters.
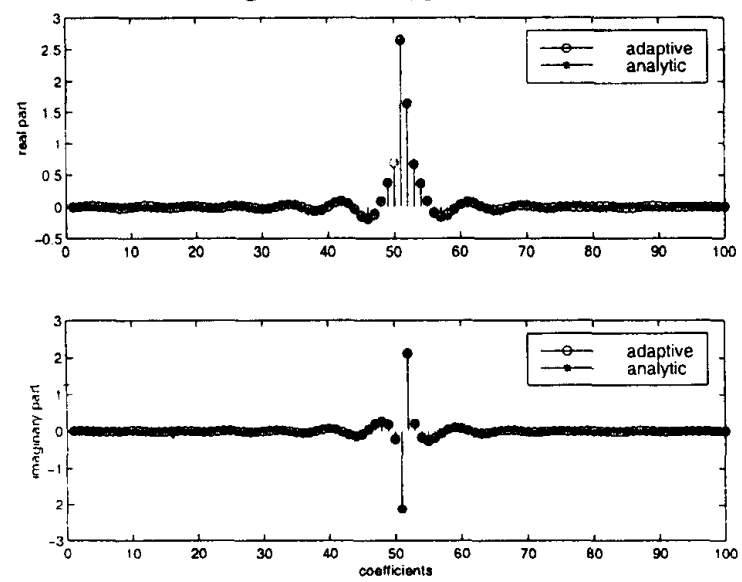

Fig. 6. Adaptive and analytic subband response for $k=0$.

\section{Conclusions}

We have introduced an analysis of an SAF system, which formulates the subband errors in dependency of LTI polyphase components only. The main result was a structural difference between what the optimum SAF requires and what the standard SAF structure provides. As a qualitative measure, this difference in structure gives alternative insight into the inaccuracies and limitations of the standard SAF approach. But as demonstrated, the approach can also be utilized to quantify errors. Different from alias measurement methods for error prediction [12], the analysis also offers access to the coefficient domain and thus allows us to state optimum SAF subband responses. As an application for the latter, an example was given that allows us to substitute the subband projection by SAF system identification with the proposed analytical polyphase approach.

\section{Acknowledgements}

The authors gratefully acknowledge Dr. Ian K. Proudler, of DERA. Malvern. UK, who partially supported this work. S. Weiss would like to thank the Royal Academy of Engineering for providing a travel grant.

\section{References}

[1] P.L. de León II and D.M. Etter. "Experimental Results with Increased Bandwidth Analysis Filters in Oversampled Subband Acoustic Echo Cancelers". IEEE Sig Proc Letters, Vol.2(No.1):pp.1-3, Jan. 1995.

[2] N.J. Fliege. Multirate Digital Signal Processing: Multirate Systems, Filter Banks, Wavelets. Wiley, 1994.

[3] A. Gilloire and M. Vetterli. "Adaptive Filtering in Subbands with Critical Sampling: Analysis, Experiments and Applications to Acoustic Echo Cancelation". IEEE Trans Signal Processing, Vol.40 (No.8):pp.1862-1875. Aug. 1992.

[4] S. Haykin. Adaptive Filter Theory. Prentice Hall, 2nd ed, 1991.

[5] W. Kellermann. "Analysis and Design of Multirate Systems for Cancellation of Acoustical Echoes". In Proc. ICASSP. vol 5, pp.2570-2573. New York, 1988.

[6] W. Kellermann. "Strategies for Combining Acoustic Echo Cancellation and Adaptive Beamforming Microphone Arrays". In Proc. IEEE ICASSP, vol I, pp.219222, Munich, April 1997.

[7] S. S. Pradhan and V. L. Reddy. "A New Approach to Subband Adaptive Filtering". IEEE Trans Signal Processing. Vol.47(No.3):pp.655-664, Warch 1999.

[8] M. Schönle, N.J. Fliege, and U. Zölzer. "Parametric Approximation of Room Impulse Responses by Multirate Systems". In Proc. IEEE ICASSP, vol I, pp.153156. Minneapolis, May 1993.

[9] P.P. Vaidyanathan. Multirate Systems and Filter Banks. Prentice Hall, 1993.

[10] S. Weiß, S.R. Dooley, R.W. Stewart, and A.K. Nandi "Adaptive Equalization in Oversampled Subbands". IEE Elec. Let., Vol.34(No.15):pp.1452-1453, July 1998.

[11] S. Weiss, R.W. Stewart, M. Schabert, I.K. Proudler, and M.W. Hoffman. "An Efficient Scheme for Broadband Adaptive Beamforming". In Asilomar Conf Sig. Sys. Comp.. Monterey, CA, Nov. 1999.

[12] S. Weiß, R.W. Stewart, A. Stenger, and R. Rabenstein. "Performance Limitations of Subband Adaptive Filters". In Proc. EUSIPCO, vol. III, pp. 1245-1248, Rodos, Sept. 1998.

[13] B. Widrow and E. Walach. Adaptive Inverse Control. Prentice Hall, 1995.

[14] R.J. Wilson, P.A. Naylor, and D. Brookes. "Performance Limitations of Subband Acoustic Echo Controllers". In Proc. IWAENC, pp.176-179, London Sept. 1997. 